\title{
Credit Name Concentration Risk: Granularity Adjustment Approximation
}

\author{
Badreddine Slime \\ Ecole Nationale de la Statistique et de l'Administration Economique (ENSAE), Paris, France \\ Email: badreddine.slime@ensae.org, badreddine.slime@gmail.com
}

How to cite this paper: Slime, B. (2016). Credit Name Concentration Risk: Granularity Adjustment Approximation. Journal ot Financial Risk Management, 5, 246-263. http://dx.doi.org/10.4236/jfrm.2016.54023

Received: October 8, 2016

Accepted: December 13, 2016

Published: December 16, 2016

Copyright $\odot 2016$ by author and Scientific Research Publishing Inc. This work is licensed under the Creative Commons Attribution International License (CC BY 4.0).

http://creativecommons.org/licenses/by/4.0/

\begin{abstract}
During the last subprime mortgage crisis, the concentration risk issue has become increasingly important in the world of finance. This risk is defined as the loss that we can incur from a large exposition of a single name counterparty, a sector or a product. This paper represents some mathematical models for assessment and quantification of the concentration risk under the Add-On approach. This study is based on the Granularity Adjustment $(G A)$. This measure quantifies the idiosyncratic risk that is neglected by the Asymptotic Single Risk Factor model ( $A S R F$ ) based on the infinitely granular assumption of the portfolio. This work is about the approximation of this measurement to simplify the formula of $G A$ using the $A d-H o c$ approach. We have implemented empirical tests to find the relation between the $G A$ and concentration indexes and we applied these results to the iBoxx portfolio.
\end{abstract}

\section{Keywords}

Credit Risk, Asymptotic Single Risk Factor, Concentration Risk, Granularity

Adjustment, Vasicek Model, Credit Risk+ Model

\section{Introduction}

The Ad-Hoc approach does not take into consideration the specific risk factors like the $P D$ and $L G D$. On the other hand, it does not allow computing the provision charge of capital requirement to cover the concentration risk. Behind this, the GA represents all specific risks neglected by the ASRF model, so it's over than the concentration risk. However, we can use it as a metric to measure this kind risk.

This paper studies the modeling and the approximation of this measure of concentration risk. We will focus on the credit environment that represents the banking book and the source of risks in the bank balance. We will restrict on the name concentration.

First, we will begin by modeling the name concentration under the granularity ad- 
justment. Next, we will implement this approach in the Vasicek and Credit Risk ${ }^{1}$ models. Then, we will suggest the approximation of the $G A$. Finally, we will implement some tests to see the efficiency of these approximations and we will use these results on the $i B o x x$ portfolio to make it available.

\section{The Formulation of Granularity Adjustment (GA)}

The $G A$ was developed to underpin the Asymptotic Single Risk Factor model ( $A S R F)$ in order to cover the idiosyncratic risk under Internal Rating Based model (IRB) of Basel II. Indeed, the $A S R F$ model supposes that the portfolio is infinitely granular and this assumption neglected the specific risk. The $G A$ formula was computed by Wilde (2001). Thereafter, Martin and Wilde (2002) used the results of Gourieroux et al. (2000) to simplify it. In this section, we will compute the $G A$ formulation under the Vasicek and Credit Risk+ models. We deem $X$ as the one-dimensional systematic factor and $L_{N}$ as the portfolio loss with $N$ loan, and giving the following notations of the mean and the variance of the conditional loss'

$$
\mu(X)=\mathbb{E}\left[L_{N} \mid X\right] \text { et } \sigma^{2}(X)=\mathbb{V}\left[L_{N} \mid X\right]
$$

For $\varepsilon=1$, the portfolio loss is equal to:

$$
L_{N}=\mu(X)+\varepsilon\left(L_{N}-\mu(X)\right)
$$

Using these notations, the $G A$ is defined as:

$$
\begin{aligned}
\operatorname{GA}_{q}\left(L_{N}\right) & =\operatorname{VaR}_{q}\left(L_{N}\right)-\operatorname{VaR}_{q}^{A S F R}\left(L_{N}\right) \\
& =\operatorname{VaR}_{q}\left(\mu(X)+\varepsilon\left(L_{N}-\mu(X)\right)\right)-\operatorname{VaR}_{q}(\mu(X))
\end{aligned}
$$

By applying the Taylor expansion on $\operatorname{VaR}_{q}\left(\mu(X)+\varepsilon\left(L_{N}-\mu(X)\right)\right)$ with second order according to the $\varepsilon=0$ and by replacing the $\varepsilon=1$, we get $^{3}$ :

$$
\begin{aligned}
& G A_{q}\left(L_{N}\right) \\
& =\left.\frac{\partial}{\partial \varepsilon} \operatorname{VaR}_{q}\left(\mu(X)+\varepsilon\left(L_{N}-\mu(X)\right)\right)\right|_{\varepsilon=0}+\left.\frac{1}{2} \frac{\partial^{2}}{\partial \varepsilon^{2}} \operatorname{VaR}_{q}\left(\mu(X)+\varepsilon\left(L_{N}-\mu(X)\right)\right)\right|_{\varepsilon=0}
\end{aligned}
$$

By computing the first and the second derivative terms, we find the following results ${ }^{4}$ :

$$
\begin{gathered}
\frac{\partial}{\partial \varepsilon} \operatorname{VaR}_{q}(X)=\mathbb{E}\left[L_{N} \mid X=\operatorname{VaR}_{q}(X)\right] \\
\frac{\partial^{2}}{\partial \varepsilon^{2}} \operatorname{VaR}_{q}(X)=-\left.\left[\frac{1}{f_{X}(x)} \frac{\partial}{\partial x}\left(f_{X}(x) \times \mathbb{V}\left[L_{N} \mid X=x\right]\right)\right]\right|_{X=V a R_{q}(X)}
\end{gathered}
$$

With $f_{X}$ defines the density function of $X$.

If we set $X=\mu(X)$, we get the following results ${ }^{5}$ :

${ }^{1}$ See Annex.

${ }^{2}$ See Annex.

${ }^{3}$ See Wilde (2001), Probing granularity, Risk Magazine, Vol 14, No 8, pp 103-106.

${ }^{4}$ See Gourieroux, Laurent, \& Scaillet (2000), Sensitivity analysis of Values at Risk, Journal of Empirical Finance.

${ }^{5}$ See Martin, \& Wilde (2002): Unsvstematic credit risk, Risk Magazine 15(11), pv 123-128. 


$$
\begin{gathered}
\frac{\partial}{\partial \varepsilon} \operatorname{VaR}_{q}(\mu(X))=0 \\
\frac{\partial^{2}}{\partial \varepsilon^{2}} \operatorname{VaR}_{q}(X)=-\left.\left[\frac{1}{2 f_{X}(x)} \times \frac{\partial}{\partial x}\left(\frac{\sigma^{2}(x) f_{X}(x)}{\mu^{\prime}(x)}\right)\right]\right|_{x=\operatorname{VaR}_{1-q}(X)}
\end{gathered}
$$

We find the general formula of $G A$ basing on these results:

$$
G A_{q}\left(L_{N}\right)=-\left.\frac{1}{2}\left[\frac{1}{\mu^{\prime}(x)}\left(\sigma^{2}(x) \frac{f_{X}^{\prime}(x)}{f_{X}(x)}+\sigma^{2}(x)^{\prime}\right)-\sigma^{2}(x) \frac{\mu^{\prime \prime}(x)}{\left(\mu^{\prime}(x)\right)^{2}}\right]\right|_{x=V a R_{1-q}(x)}
$$

Therefore, if we want to explain this formula we should use a risk model. The most prevalent models for the banking book to calculate the capital request for the credit risk is: The Vasicek and the Credit Risk+ models. The first one is deemed as a structural model, and the second one belongs to the intensity model. In the following paragraphs, we will develop the $G A$ formula under these models.

\section{- The $G A$ formula under the Vasicek model:}

The Vasicek $^{6}$ model supposes that the systematic factor is following the Gaussian distribution $X \sim N(0,1)$, and this result leads to:

$$
\frac{f_{X}^{\prime}(x)}{f_{X}(x)}=-x
$$

Substituting in the formula of $G A$, we get:

$$
\begin{aligned}
G A_{q}^{\text {Vasicek }}\left(L_{N}\right) & =\left.\frac{1}{2}\left[\frac{1}{\mu^{\prime}(x)}\left(x \sigma^{2}(x)-\sigma^{2}(x)^{\prime}\right)+\sigma^{2}(x) \frac{\mu^{\prime \prime}(x)}{\left(\mu^{\prime}(x)\right)^{2}}\right]\right|_{x=\Phi^{-1}(1-q)} \\
& =\left.\frac{1}{2 \mu^{\prime}(x)}\left[\left(x+\frac{\mu^{\prime \prime}(x)}{\mu^{\prime}(x)}\right) \sigma^{2}(x)-\sigma^{2}(x)^{\prime}\right]\right|_{x=\Phi^{-1}(1-q)}
\end{aligned}
$$

Thus, we can compute the components that allow computing the $G A^{7}$ :

$$
\begin{gathered}
\mu(x)=\mathbb{E}\left[L_{N} \mid x\right]=\sum_{i=1}^{N} s_{i} \mu_{i}(x)=\sum_{i=1}^{N} s_{i} \times \mathbb{E}\left[L G D_{i}\right] \times P D_{i}(x) \\
\sigma^{2}(x)=\mathbb{V}\left[L_{N} \mid x\right]=\sum_{i=1}^{N} s_{i}^{2} \sigma_{i}^{2}(x)
\end{gathered}
$$

with $\mu_{i}(x)=\mathbb{E}\left[L G D_{i}\right] \times P D_{i}(x)=\mathbb{E}\left[L G D_{i}\right] \times \Phi\left(\frac{\Phi^{-1}\left(P D_{i}\right)-\sqrt{\rho_{i}} x}{\sqrt{1-\rho_{i}}}\right)$

$$
\Rightarrow \mu_{i}^{\prime}(x)=-\mathbb{E}\left[L G D_{i}\right] \times \sqrt{\frac{\rho_{i}}{1-\rho_{i}}} \times \varphi\left(\frac{\Phi^{-1}\left(P D_{i}\right)-\sqrt{\rho_{i}} x}{\sqrt{1-\rho_{i}}}\right)
$$

and $\mu_{i}^{\prime \prime}(x)=\sqrt{\frac{\rho_{i}}{1-\rho_{i}}} \times\left(\frac{\Phi^{-1}\left(P D_{i}\right)-\sqrt{\rho_{i}} x}{\sqrt{1-\rho_{i}}}\right) \times \mu_{i}^{\prime}(x)$

${ }^{6}$ See Vasicek (1987). Probability of loss on loan portfolio, KMV Corporation, San Francisco, USA. ${ }^{7}$ See Annex. 
We have also:

$$
\begin{aligned}
\sigma_{i}^{2}(x) & =\mathbb{V}\left[L_{i} \mid x\right]=\mathbb{E}\left[\left(L G D_{i} \times D_{i}\right)^{2} \mid x\right]-\left(\mathbb{E}\left[L G D_{i} \times D_{i} \mid x\right]\right)^{2} \\
& =\mathbb{E}\left[\left(L G D_{i}\right)^{2}\right] \times \mathbb{E}\left[\left(D_{i}\right)^{2} \mid x\right]-\left(\mathbb{E}\left[L G D_{i}\right]\right)^{2} \times\left(\mathbb{E}\left[D_{i} \mid x\right]\right)^{2} \\
& =\left(\left(\mathbb{V}\left[L G D_{i}\right]+\left(\mathbb{E}\left[L G D_{i}\right]\right)^{2}\right)-\left(\mathbb{E}\left[L G D_{i}\right]\right)^{2} \times P D_{i}(x)\right) \times P D_{i}(x) \\
& =C_{i} \mu_{i}(x)-\mu_{i}(x)^{2}
\end{aligned}
$$

With $C_{i}=\frac{\mathbb{E}\left[L G D_{i}\right]^{2}+\mathbb{V}\left[L G D_{i}\right]}{\mathbb{E}\left[L G D_{i}\right]}$ and $D_{i}$ is the default variable ${ }^{8}$.

The derivative function regarding to $x$ is equal to:

$$
\begin{aligned}
& \mu^{\prime}(x)=\sum_{i=1}^{N} s_{i} \times \mu_{i}^{\prime}(x), \mu^{\prime \prime}(x)=\sum_{i=1}^{N} s_{i} \times \sqrt{\frac{\rho_{i}}{1-\rho_{i}}} \times\left(\frac{\Phi^{-1}\left(P D_{i}\right)-\sqrt{\rho_{i}} x}{\sqrt{1-\rho_{i}}}\right) \times \mu_{i}^{\prime}(x) \\
& \sigma^{2}(x)=\sum_{i=1}^{N} s_{i}^{2} \times \mu_{i}(x) \times\left(C_{i}-\mu_{i}(x)\right), \sigma^{2}(x)^{\prime}=\sum_{i=1}^{N} s_{i}^{2} \times \mu_{i}^{\prime}(x) \times\left(C_{i}-2 \mu_{i}(x)\right)
\end{aligned}
$$

By developing the $G A$ under $x=\Phi^{-1}(1-q)$, we find the following formula:

$$
\begin{aligned}
G A_{q}^{\text {Vasicek }}\left(L_{N}\right)= & \frac{1}{2 \mu^{\prime}\left(\Phi^{-1}(1-q)\right)}\left[\sum_{i=1}^{N} s_{i}^{2} \times \mu_{i}\left(\Phi^{-1}(1-q)\right) \times\left(\delta\left(C_{i}-\mu_{i}\left(\Phi^{-1}(1-q)\right)\right)\right.\right. \\
& \left.-\frac{\mu_{i}^{\prime}\left(\Phi^{-1}(1-q)\right)}{\mu_{i}\left(\Phi^{-1}(1-q)\right)} \times\left(C_{i}-2 \mu_{i}\left(\Phi^{-1}(1-q)\right)\right)\right]
\end{aligned}
$$

With $\delta=\left(\Phi^{-1}(1-q)+\frac{\mu^{\prime \prime}\left(\Phi^{-1}(1-q)\right)}{\mu^{\prime}\left(\Phi^{-1}(1-q)\right)}\right)$

\section{- The $G A$ formula under the Credit Risk+ model:}

As we have seen to compute the GA formula, we need to calculate the following quantities $\mu(x), \sigma^{2}(x)$, and $f_{X}(x)$ that depend to the model. The assumption of the Credit Risk $t^{9}$ model is that $X \sim \Gamma(\alpha, \beta)$ where $\alpha=\frac{1}{\beta}$. Then, we obtain the following relation:

$$
\frac{f_{X}^{\prime}(x)}{f_{X}(x)}=(\alpha-1) x-1
$$

We can explain the $G A$ formula by computing the following components:

$$
\mu(x)=\sum_{i=1}^{N} s_{i} \mu_{i}(x), \sigma^{2}(x)=\sum_{i=1}^{N} s_{i}^{2} \sigma_{i}^{2}(x)
$$

The expression of $\mu_{i}(x)$ is given by ${ }^{10}$ :

$$
\begin{aligned}
\mu_{i}(x)= & \mathbb{E}\left[L G D_{i}\right] \times P D_{i}(x)=\mathbb{E}\left[L G D_{i}\right] \times P D_{i} \times\left(1-w_{i}+w_{i} \times x\right) \\
& \Rightarrow \mu(x)=\sum_{i=1}^{N} \mathbb{E}\left[L G D_{i}\right] \times P D_{i} \times\left(1-w_{i}+w_{i} \times x\right),
\end{aligned}
$$

${ }^{8}$ See Annex.

${ }^{9}$ See Credit Suisse Financial Products (1997). Credit Riskt: A Credit Risk Management Framework. London, 1997.

${ }^{10}$ See Annex. 
And $\mu^{\prime}(x)=\sum_{i=1}^{N} \mathbb{E}\left[L G D_{i}\right] \times P D_{i} \times w_{i}$ et $\mu^{\prime \prime}(x)=0$

We have for the conditional variance:

$$
\begin{aligned}
\sigma_{i}^{2}(x) & =\mathbb{E}\left[\left(L G D_{i} \times D_{i}\right)^{2} \mid x\right]-\left(\mathbb{E}\left[L G D_{i} \times D_{i} \mid x\right]\right)^{2} \\
& =\mathbb{E}\left[\left(L G D_{i}\right)^{2}\right] \times \mathbb{E}\left[\left(D_{i}\right)^{2} \mid x\right]-\left(\mathbb{E}\left[L G D_{i}\right]\right)^{2} \times\left(P D_{i}(x)\right)^{2} \\
& =\mathbb{E}\left[\left(L G D_{i}\right)^{2}\right] \times \mathbb{E}\left[\left(D_{i}\right)^{2} \mid x\right]-\left(\mu_{i}(x)\right)^{2} \\
& =C_{i} \mu_{i}(x)+\left(\mu_{i}(x)\right)^{2} \times \frac{\mathbb{V}\left[L G D_{i}\right]}{\mathbb{E}\left[L G D_{i}\right]^{2}}
\end{aligned}
$$

With $C_{i}=\frac{\mathbb{E}\left[L G D_{i}\right]^{2}+\mathbb{V}\left[L G D_{i}\right]}{\mathbb{E}\left[L G D_{i}\right]}$

We conclude that:

$$
\sigma^{2}(x)=\sum_{i=1}^{N} s_{i}^{2} \mu_{i}(x) \times\left(C_{i}+\mu_{i}(x) \times \frac{\mathbb{V}\left[L G D_{i}\right]}{\mathbb{E}\left[L G D_{i}\right]^{2}}\right)
$$

Therefore, we have:

$$
\sigma^{2}(x)^{\prime}=\sum_{i=1}^{N} s_{i}^{2} \mu_{i}^{\prime}(x) \times\left(C_{i}+2 \mu_{i}(x) \times \frac{\mathbb{V}\left[L G D_{i}\right]}{\mathbb{E}\left[L G D_{i}\right]^{2}}\right)
$$

These results we lead us to the $G A$ formulation found it by Gordy and Lutkebohmert $(2007)^{11}$ :

$$
\begin{aligned}
G A_{q}^{C R+}\left(L_{N}\right)= & \frac{1}{2 U L} \sum_{i=1}^{N} s_{i}^{2}\left[\left(\delta C_{i} \times\left(U L_{i}+E L_{i}\right)+\delta\left(U L_{i}+E L_{i}\right)^{2} \times \frac{\mathbb{V}\left[L G D_{i}\right]}{\mathbb{E}\left[L G D_{i}\right]^{2}}\right)\right. \\
& \left.-U L_{i} \times\left(C_{i}+2\left(U L_{i}+E L_{i}\right) \times \frac{\mathbb{V}\left[L G D_{i}\right]}{\mathbb{E}\left[L G D_{i}\right]^{2}}\right)\right]
\end{aligned}
$$

With,

$$
\begin{aligned}
& \qquad E L_{i}=\mathbb{E}\left[L G D_{i}\right] \times P D_{i}, U L_{i}=\mathbb{E}\left[L G D_{i}\right] \times P D_{i} \times w_{i} \times\left(\operatorname{VaR}_{q}(X)-1\right), U L=\sum_{i=1}^{N} s_{i} U L_{i} \\
& \text { And } \delta=\left(\operatorname{VaR}_{q}(X)-1\right) \times\left(\alpha+\frac{1-\alpha}{\operatorname{VaR}_{q}(X)}\right)
\end{aligned}
$$

\section{The Granularity Adjustment Approximation}

The aim of this study is the implementation of algorithmic tests to test approximations of $G A$. These algorithmic tests will be established on $\mathrm{R}$ and under the following assumptions:

\footnotetext{
${ }^{11}$ See Gordy, \& Lutkebohmert (2007), Granularity adjustment for Basel II, Discussion Paper Series 2: Banking and Financial Studies, Deutsche Bundesbank (1).

See Gordy, \& Lutkebohmert (2013), Granularity Adjustment for Regulatory Capital Assessment, International Journal of Central Banking.

See Lutkebohmert (2009). Concentration Risk in Credit Portfolios. Springer.
} 
- The HKI (Hannah-Kay Index) parameter is equal to 3.

- The HIS (Hammami-Slime Index) parameter is equal to 0.25.

- The generation of exposures follows the Log-normal distribution.

- The parameter of the Gamma distribution is equal 0.31 .

- The quantile is equal to $99.9 \%$.

\subsection{The Reduced Form of GA}

The authors of the $G A$ formula below the Credit Risk+ suggest a simplification under the assumption that quantities of $E L_{i}$ and $U L_{i}$ are enough small. So, we can neglect $\left(U L_{i}+E L_{i}\right)^{2} \approx 0$ and $U L_{i} \times\left(U L_{i}+E L_{i}\right) \approx 0$. The simplified $G A$ becomes:

$$
\widetilde{G A}_{q}^{C R+}\left(L_{N}\right) \approx \frac{1}{2 U L} \sum_{i=1}^{N} s_{i}^{2} \times C_{i} \times\left(\delta\left(U L_{i}+E L_{i}\right)-U L_{i}\right)
$$

By the same way, we can approximate this formula below the Vasicek model giving the assumption $\mu_{i}\left(\Phi^{-1}(1-q)\right)^{2} \approx 0$ and $\mu_{i}\left(\Phi^{-1}(1-q)\right) \times \mu_{i}^{\prime}\left(\Phi^{-1}(1-q)\right) \approx 0$, by:

$$
\widetilde{G A}_{q}^{\text {Vasicek }}\left(L_{N}\right) \approx \frac{1}{2 \mu^{\prime}\left(\Phi^{-1}(1-q)\right)}\left[\sum_{i=1}^{N} s_{i}^{2} \times C_{i} \times\left(\delta \times \mu_{i}\left(\Phi^{-1}(1-q)\right)-\mu_{i}^{\prime}\left(\Phi^{-1}(1-q)\right)\right)\right]
$$

This test allows verifying the validity of these approximate formulas of $G A$. The $\mathrm{Ta}$ ble 1 summarizes the formulations under the both models Vasicek and Credit Riskt.

The test implementation is based on portfolio generating of some $N=1000$ exposures according to the Log-normal distribution. Then, we compute the full and the approximate $G A$ under the both models Vasicek and Credit Riskt. We repeat this operation one thousand times to get 1000 portfolios at the end. Test steps are described on the following algorithm:

1) Generate 1000 exposures according to the Log-normal $(10,3)$ distribution.

2) Generate 1000 probabilities of default according to the uniform distribution.

3) Generate 1000 correlation coefficient according to the uniform distribution between 0.12 and 0.24 .

4) Compute the full GA according to the two models.

5) Compute the approximate GA according to the two models.

Table 1. Summary of the $G A$ formula depending on model.

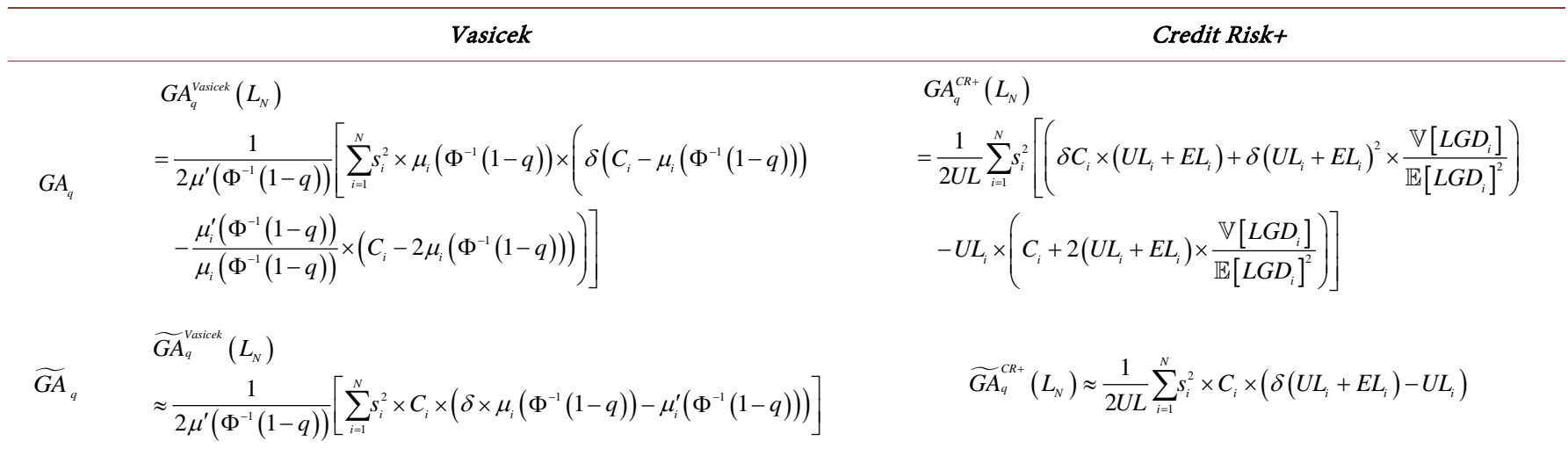


6) Iterate 1000 times the steps from 1 to 5 .

7) Statistical test of the average under the generated data of the full and the approximate GA.

8) Statistical test of the variance homogeneity under the generated data of the full and the approximate GA.

This test allows us to determine the conditions of using the approximate $G A$ in order to simplify computing. First, we get in the Vasicek model with an interval of default probabilities between 0 and $1 \%$. We conclude that the two values are very close. Furthermore, the Student test of the average and the Fisher test of the variance are conclusive and we find respectively a p-value equal to $24 \%$ and $5.5 \%$. This result underpins the approximate formula of the $G A$. On the other hand, if we have the un-conditional default probabilities go beyond of $1 \%$ then this approximation doesn't more work. The Figure 1 reproduces the results of this test:

In regards to the Credit Risk+ model, we can prove using tests that the approximation formula of $G A$ still suitable when the probabilities of default are between 0 and $10 \%$. We get in by the same way and we generate the $P D s$ between 0 and $10 \%$. The Student test on the average and the Fisher test on the variance give respectively a p-value of $49 \%$ and de $16 \%$. On the other side, this result is no more suitable for the PDs beyond of $10 \%$. As conclusion, the condition that makes the approximation formula suitable for Vasicek model is the PDs portfolio between $0 \%$ and 1\%, and for the Credit Risk+ model is the PDs portfolio between $0 \%$ and $10 \%$. The Figure 2 shows the evolution of the full and the approximate $G A$.

\subsection{The Regression of GA on the Concentration Indexes}

- The regression of the GA on the Herfindahl-Hirschman Index (HHI):

We find into the $G A$ formula the square of shares $s_{i}^{2}$, and these represent compo-
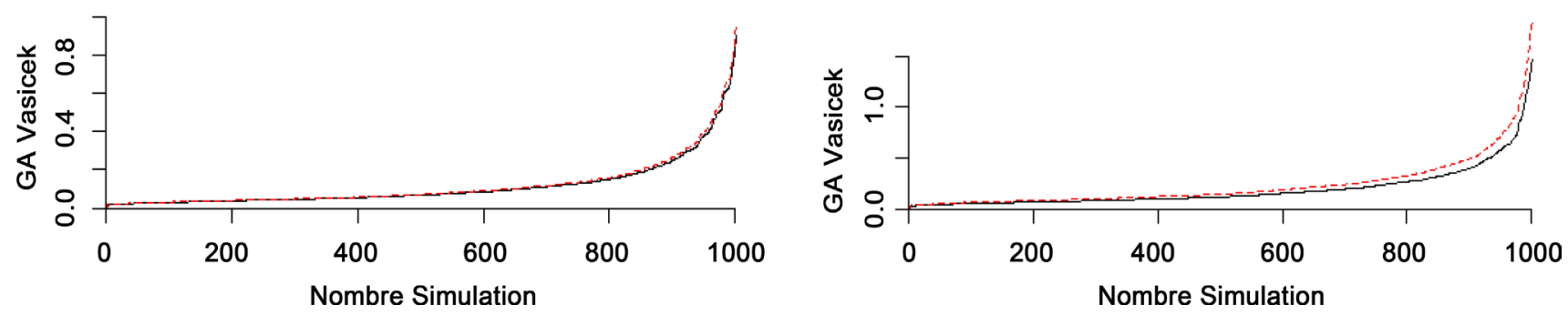

Figure 1. The evolution of the $G A$ under the Vasicek Model according to number of simulations.
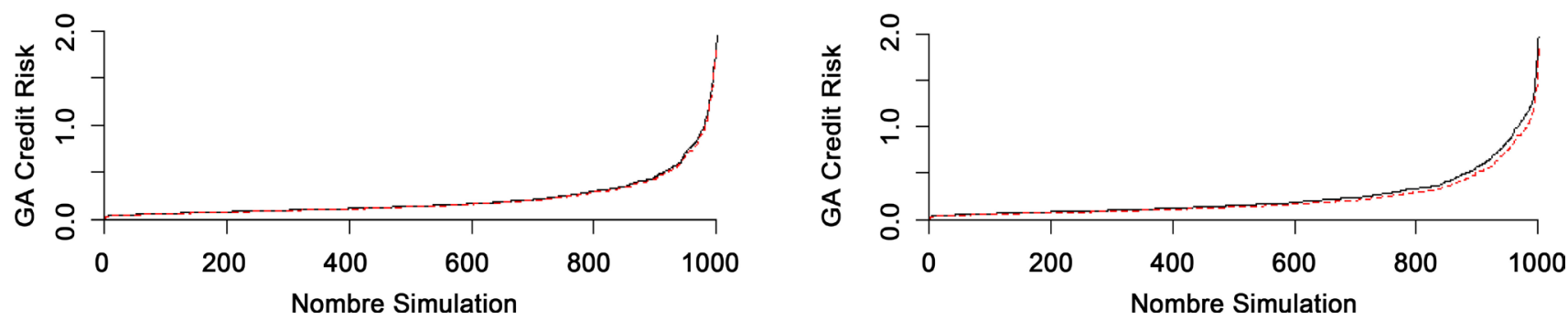

Figure 2. The evolution of the $G A$ under the Credit Risk+ Model according to number of simulations. 
nents of the $H H I^{2}$ index. Furthermore, in the case of a homogeneous portfolio regarding to specific risk factors, we get a linear relation between the $G A$ and the $H H I$ :

$$
\left\{\begin{aligned}
& G A_{q}^{\text {Vasicek }}\left(L_{N}\right)= \frac{\mu\left(\Phi^{-1}(1-q)\right)}{2 \mu^{\prime}\left(\Phi^{-1}(1-q)\right)} \times\left[\delta\left(C-\mu\left(\Phi^{-1}(1-q)\right)\right)\right. \\
&\left.-\frac{\mu^{\prime}\left(\Phi^{-1}(1-q)\right)}{\mu\left(\Phi^{-1}(1-q)\right)} \times\left(C-2 \mu\left(\Phi^{-1}(1-q)\right)\right)\right] \times H H I \\
& G A_{q}^{C R+}\left(L_{N}\right)= \frac{1}{2 U L} \times\left[\left(\delta C \times(U L+E L)+\delta(U L+E L)^{2} \times \frac{\mathbb{V}[L G D]}{\mathbb{E}[L G D]^{2}}\right)\right. \\
&-U L \times\left(C+2(U L+E L) \times \frac{\mathbb{V}[L G D]}{\left.\left.\mathbb{E}[L G D]^{2}\right)\right] \times H H I}\right) \\
& \Rightarrow\left\{\begin{array}{l}
G A_{q}^{\text {Vasicek }}\left(L_{N}\right)=\text { Coeff }(P D, L G D, q) \times H H I \\
G A_{q}^{C R+}\left(L_{N}\right)=\text { Coeff }(P D, L G D, w, q) \times H H I
\end{array}\right.
\end{aligned}\right.
$$

where $H H I=\sum_{i=1}^{N} s_{i}^{2}$.

The Figure 3 shows the evolution of the $G A$ according to the $H H I$ index in the case of homogenous portfolios ( $P D=5 \%, L G D=45 \%, w=12 \%$ ):

The goal of this test is to verify the validity of this relation on the non-homogeneous portfolio. For this, we establish the following test:

1) Generate 1000 exposures according to the $\log$-normal $(10,3)$ distribution.

2) Generate 1000 probabilities of default according to the uniform distribution (5\%, $10 \%)$.

3) Generate 1000 correlation coefficient according to the uniform distribution between 0.12 and 0.24 .

4) Compute the full $G A$ according to the two models (Vasicek and Credit Risk+).

5) Compute the $H H I$ index.

6) Iterate 1000 times the steps from 1 to 5.

7) Apply the linear regression under the simulated $G A$ according to the simulated HHI.

If we take an interval of $P D s$ between $0 \%$ and $20 \%$, we obtain the following results in the Figure 4.

The Table 2 summarizes the characteristics of the linear regression.

From these results, we can deduce that the relationship of linearity between the $G A$ and the $H H I$ remains valid for minimum concentrations. Otherwise, you can have quite substantial dispersions around the regression for fairly major indexes.

\section{- The regression of the GA on the Hannah-Kay Index (HKI):}

We couldn't find directly the relation between the $G A$ and the $H K I$ even though in case of a homogeneous portfolio. Therefore, we will use an empirical approach to get this relation. The $H K I^{13}$ is defined by:

\footnotetext{
${ }^{12}$ See Herfindahl (1950). Concentration in the U.S. Steel Industry, Dissertation, Columbia University. See Hirschmann (1964). The paternity of an index. American Economic Review, 54, 5, pp. 761.

${ }^{13}$ See Hannah, \& Kay (1977). Concentration in modern industry. Mac Millan Press, London.
} 

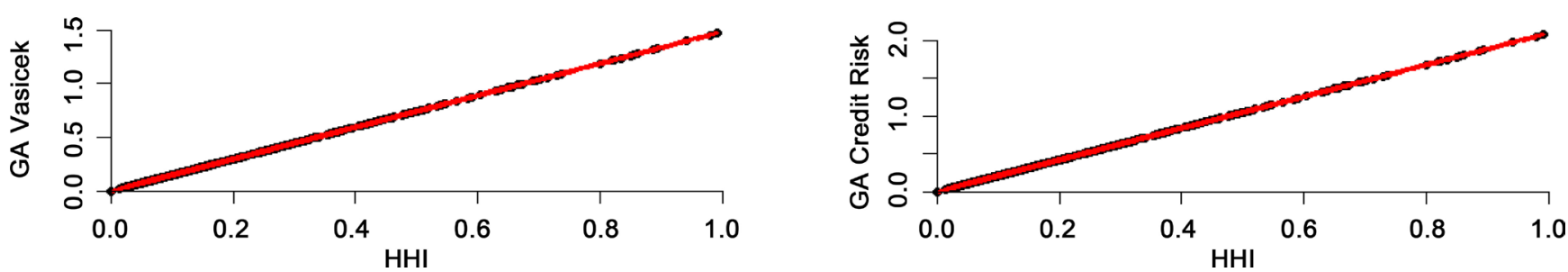

Figure 3. The evolution of GA regarding to HHI in case of a homogeneous portfolio.
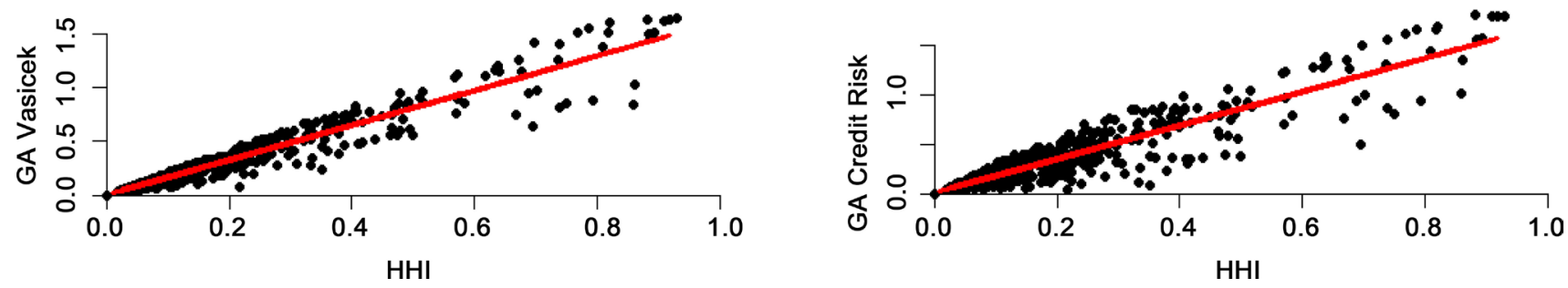

Figure 4. The evolution of GA regarding to HHI with $P D \in[0,20 \%]$.

Table 2. Summary of linear regression of $G A$ on $H H I$.

\begin{tabular}{cccc}
\hline & Coefficient & Standard Residue & R-Squared \\
\hline GA Vasicek & 1.61 & 0.065 & 0.93 \\
GA Credit Risk+ & 1.70 & 0.1 & 0.87 \\
\hline
\end{tabular}

$$
H K I=\left(\sum_{i=1}^{N} s_{i}^{\alpha}\right)^{\frac{1}{(\alpha-1)}} \text { avec } \alpha>0 \text { et } \alpha \neq 1
$$

Basing on the empirical experience, we get a non-linear regression relation:

$$
\Rightarrow\left\{\begin{array}{l}
G A_{q}^{\text {Vasicek }}\left(L_{N}\right)=a_{1}^{V} \times H K I^{\frac{\alpha-1}{\alpha}}+a_{2}^{V} \times H K I^{\frac{2 \times(\alpha-1)}{\alpha}} \\
G A_{q}^{C R+}\left(L_{N}\right)=a_{1}^{C R} \times H K I^{\frac{\alpha-1}{\alpha}}+a_{2}^{C R} \times H K I^{\frac{2 \times(\alpha-1)}{\alpha}}
\end{array} \text { With } \alpha\right. \text { is the HKI parameter }
$$

We process in the same way to the last implementation. Indeed, we generate $N=1000$ exposures with the Log-normal and we compute the GA and the HKI index. The description of the algorithm steps is:

1) Generate 1000 exposures according to the Log-normal (10, 3) distribution.

2) Generate 1000 probabilities of default according to the uniform distribution (5\%,10\%).

3) Generate 1000 correlation coefficient according to the uniform distribution between 0.12 and 0.24 .

4) Compute the full GA according to the two models (Vasicek and Credit Riskt).

5) Compute the HKI index.

6) Iterate 1000 times the steps from 1 to 5 .

7) Apply the nonlinear regression under the simulated GA according to the simulated HKI.

In the case of homogenous portfolios, the Figure 5 shows the evolution of the GA according to the HKI index, and coefficients of the non-linear regression are respectively $a_{1}^{V}=0.199, a_{2}^{V}=1.236$ and $a_{1}^{C R}=0.282, a_{2}^{C R}=1.748 \quad(P D=5 \%, L G D=45 \%$, $w=12 \%)$ : 

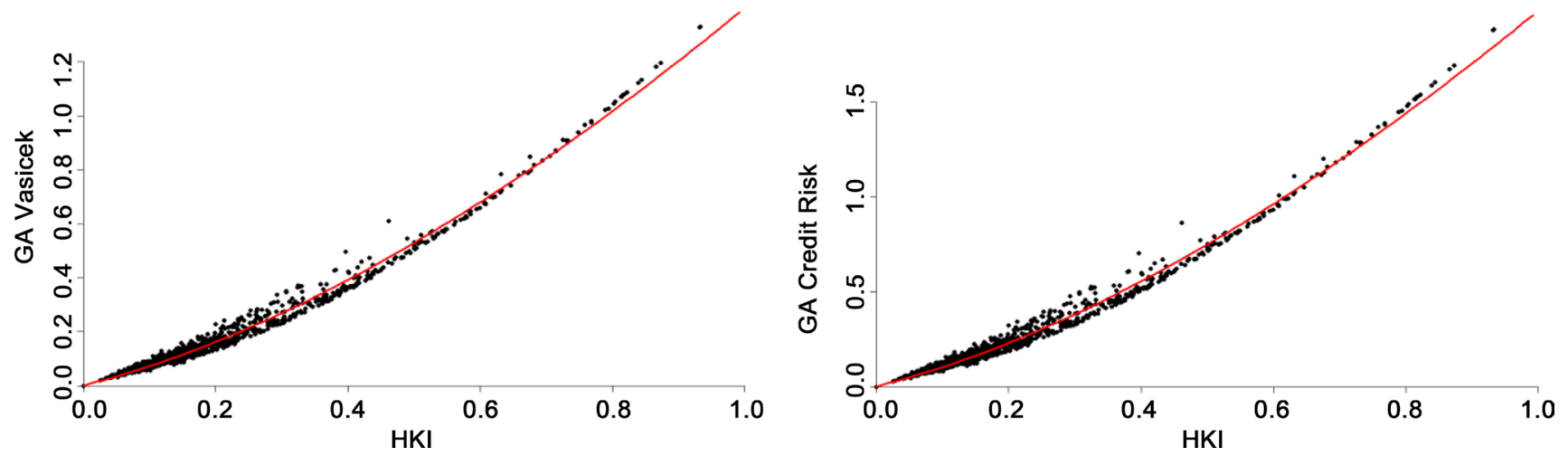

Figure 5. The evolution of $G A$ regarding to $H K I$ in case of a homogeneous portfolio.
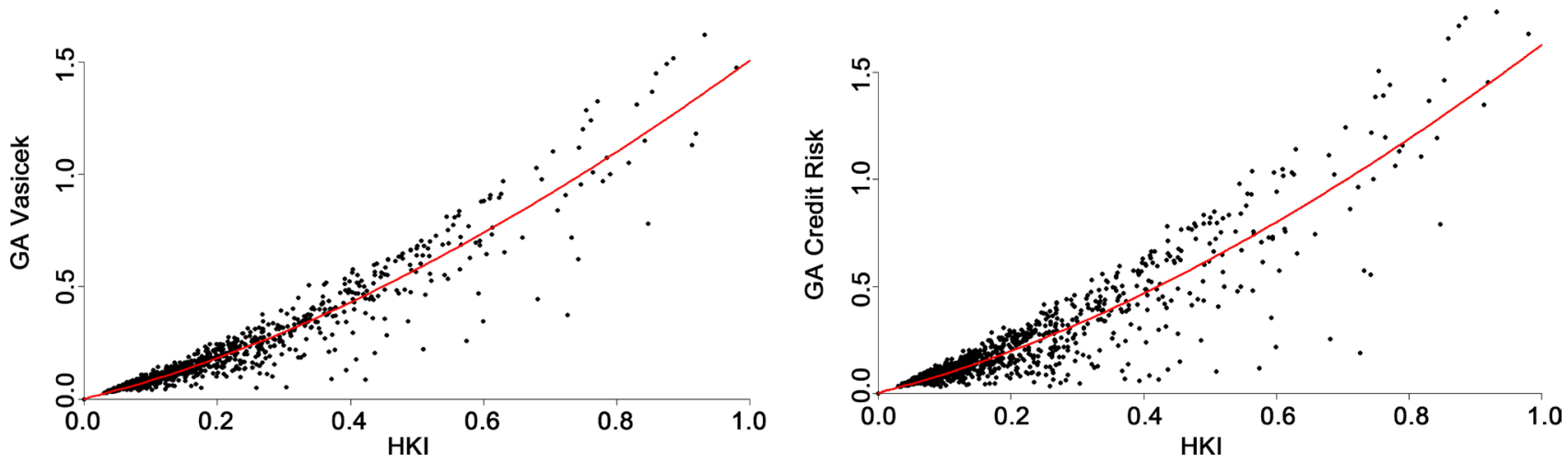

Figure 6. The evolution of GA regarding to HKI with $P D \in[0,20 \%]$.

If we take an interval of PDs between $0 \%$ and $20 \%$, we obtain the following results in the Figure 6.

We can conclude that this relationship between the $G A$ and the $H K I$ remains valid for minimum concentrations. Otherwise, you can have quite substantial dispersions around the regression for fairly major indexes.

- The regression of the GA on The Hammami-Slime Index (HSD):

We can't directly find the relation between GA and HSI even though in case of a homogeneous portfolio. Therefore, we will use an empirical approach to get this relation. The $\mathrm{HSI}^{14}$ is defined by:

$$
H S I=\sum_{i=1}^{N} s_{i}^{1+\alpha} ; 0<\alpha \leq 1
$$

Using the empirical study, we get a non-linear regression relation:

$$
\Rightarrow\left\{\begin{array}{l}
G A_{q}^{\text {Vasicek }}\left(L_{N}\right)=a_{1}^{V} \times H S I^{\frac{1}{\alpha}}+a_{2}^{V} \times H S I^{\frac{(\alpha+1)}{\alpha}} \\
G A_{q}^{C R+}\left(L_{N}\right)=a_{1}^{C R} \times H S I^{\frac{1}{\alpha}}+a_{2}^{C R} \times H S I^{\frac{(\alpha+1)}{\alpha}}
\end{array} \text { With } \alpha\right. \text { is the HSI parameter }
$$

We process in the same way to the last implementation. Indeed, we generate $N=1000$ exposures with the Log-normal and we compute the $G A$ and the $H S I$ index. The description of the algorithm steps is:

\footnotetext{
${ }^{14}$ See Slime, \& Hammami (2016). Concentration Risk: The Comparison of the Ad-Hoc Approach Indexes. Journal of Financial Risk Management, 5, 43-56.
} 
1) Generate 1000 exposures according to the $\log$-normal $(10,3)$ distribution.

2) Generate 1000 probabilities of default according to the uniform distribution (5\%, $10 \%)$.

3) Generate 1000 correlation coefficient according to the uniform distribution between 0.12 and 0.24 .

4) Compute the full GA according to the two models (Vasicek and Credit Riskt).

5) Compute the HSI index.

6) Iterate 1000 times the steps from 1 to 5.

7) Apply the nonlinear regression under the simulated GA according to the simulated HSI.

In the case of homogenous portfolios, the Figure 7 shows the evolution of the $G A$ according to the HSI index, and the coefficients of the non-linear regression are respectively $a_{1}^{V}=4.7, a_{2}^{V}=-3.17$ and $a_{1}^{C R}=6.65, a_{2}^{C R}=-4.49 \quad(P D=5 \%, L G D=45 \%$, $w=12 \%)$.

If we take an interval of $P D s$ between $0 \%$ and $20 \%$, we obtain the following results in the Figure 8.

We can conclude that this relationship between the $G A$ and the $H S I$ remains valid for minimum concentrations. Otherwise, you can have quite substantial dispersions around the regression for fairly major indexes.
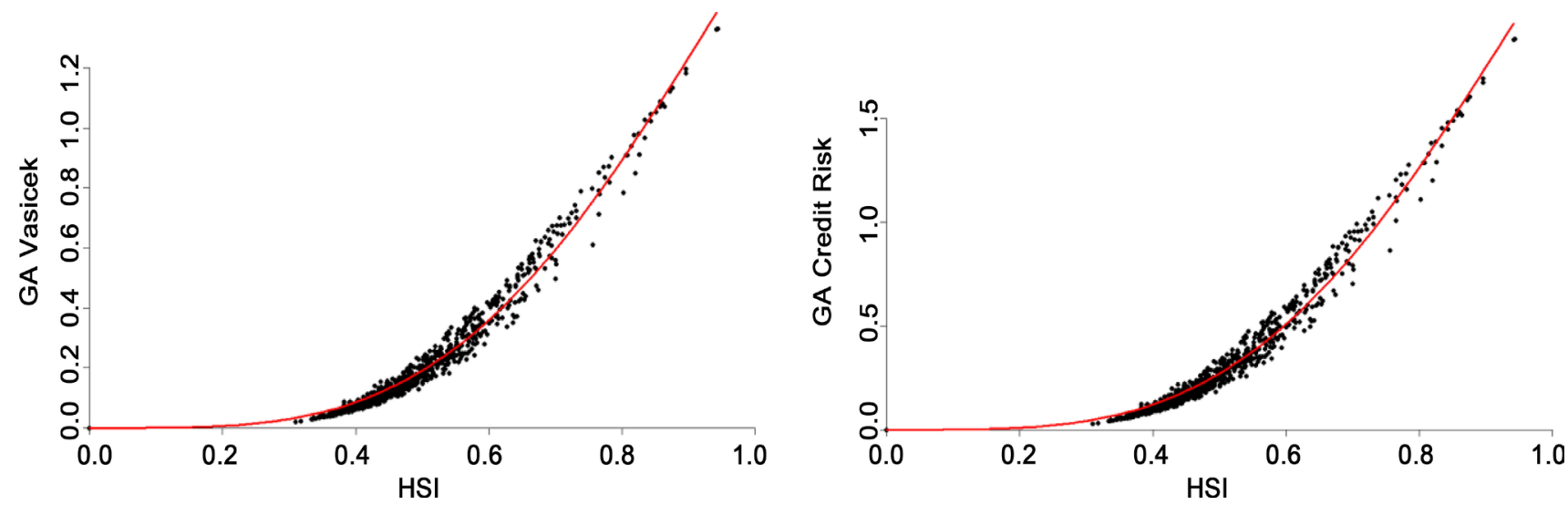

Figure 7. The evolution of $G A$ regarding to $H S I$ in case of a homogeneous portfolio.
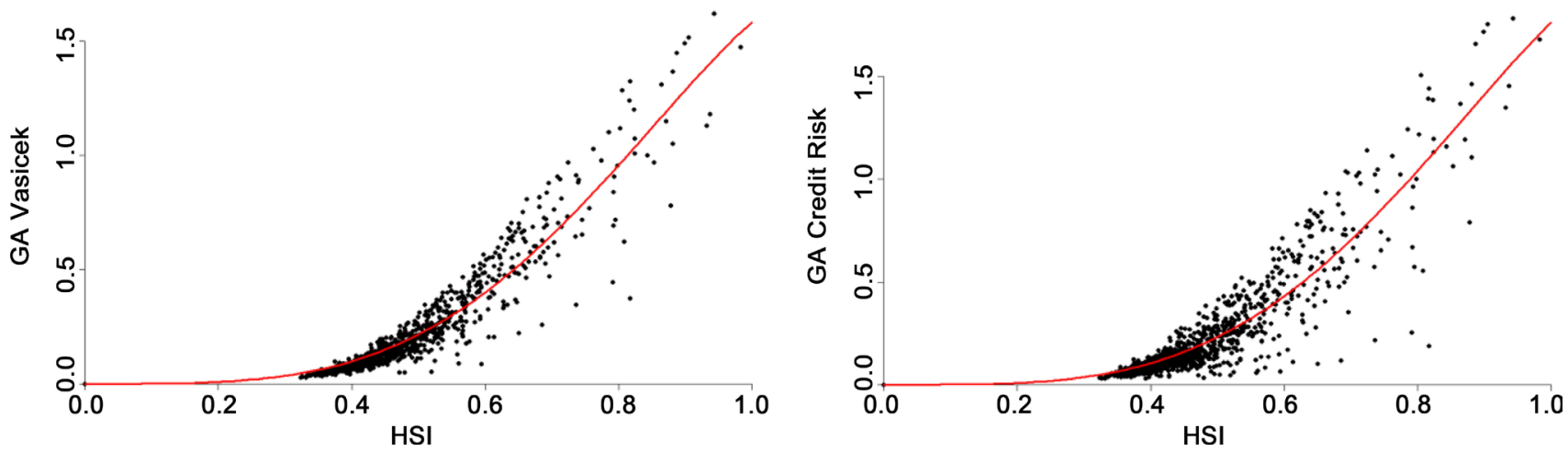

Figure 8. The evolution of $G A$ regarding to $H S I$ with $P D \in[0,20 \%]$. 


\section{Application: iBoxx Portfolio}

In this section, we will apply the obtained results under an iBoox portfolio. We will build some portfolios given the composition of this index. We will deem that the portfolio building this index is the market portfolio. The $i$ Boox contains 1663 exposures over 10 sectors and 36 countries. The total amount of debt is 1 trillion Euros. The Figure 9 and Figure 10 show repartitions by sector and by countries (the displayed data are dated 30/06/2015).

We can also have the repartition by rating in the Figure 11 .

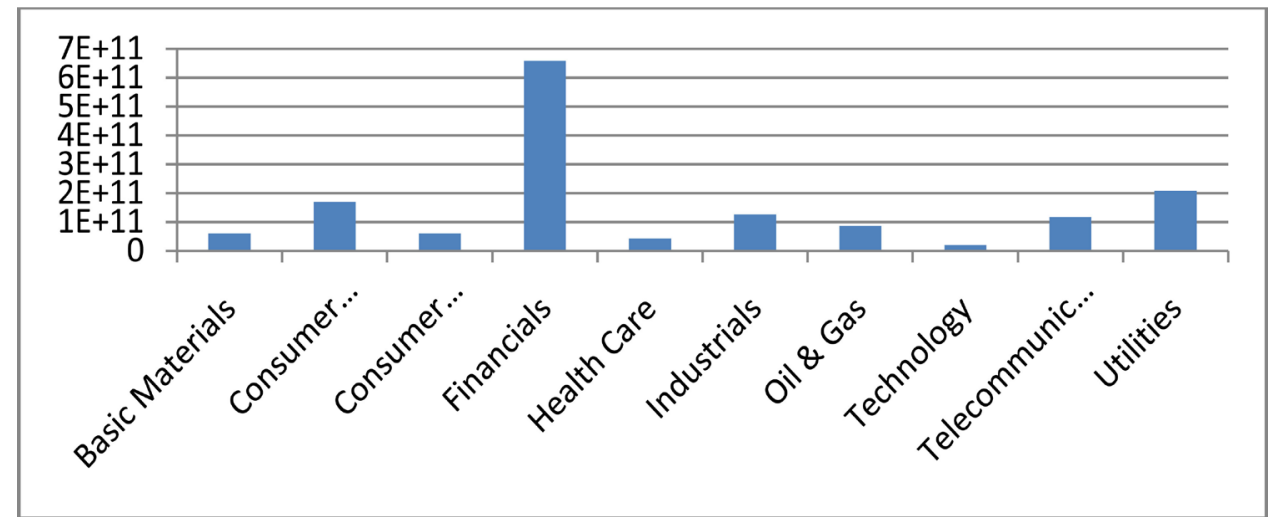

Figure 9. Graph of exposures by sector.

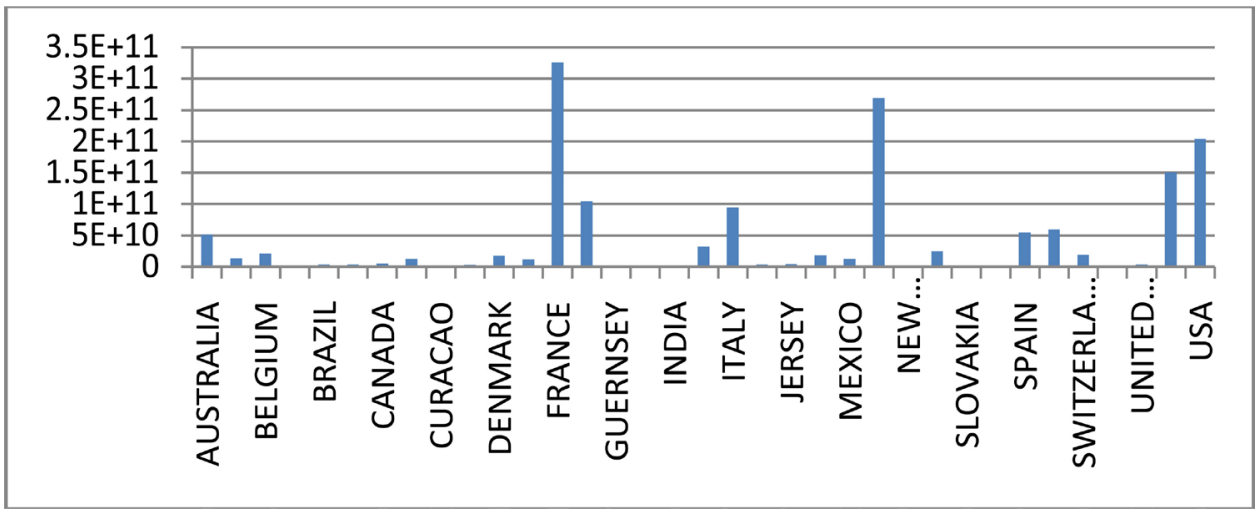

Figure 10. Graph of exposures by countries.

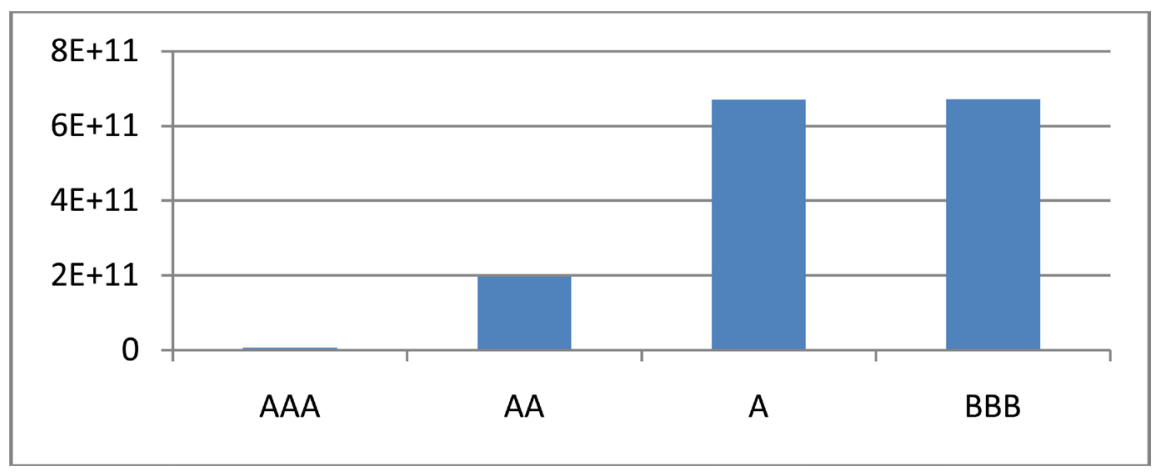

Figure 11. Graph of exposures by rating. 
The Table 3 displays the mapping between the probabilities of default and the rating ${ }^{15}$.

Firstly, we can study the concentration of the iBoxx portfolio to get a global view of the concentration. The Lorenz curve, in the Figure 12, allows us to have the dispersion of exposures by counterparty.

Basing on the graph, we have an almost equal distribution between exposures. We can make a first feeling that the name concentration is small. Therefore, we use also the other metrics to confirm this conclusion. Indeed, we compute the tree concentration indexes and the $G A$. The Table 4 summarizes the result compute of these metrics.

Giving these results, we can conclude that the name concentration is neglected.

After this study, we will take a small portfolio with 100 exposures to see the impact of the number of exposures on the name concentration under these metrics. For this, we will do a random selection from the iBoxx composition. We can use regressions of the GA on concentration indexes to compute the name concentration risk. We use the same algorithms in the third section. The Figure 13 below shows the simulation result.

The Table 5 summarizes the obtained results:

Table 3. The mapping table between the rating and the PDs.

\begin{tabular}{cc}
\hline Rating & $P D$ \\
\hline AAA & $0.09 \%$ \\
AA & $0.20 \%$ \\
A & $0.75 \%$ \\
BBB & $2.02 \%$ \\
\hline
\end{tabular}

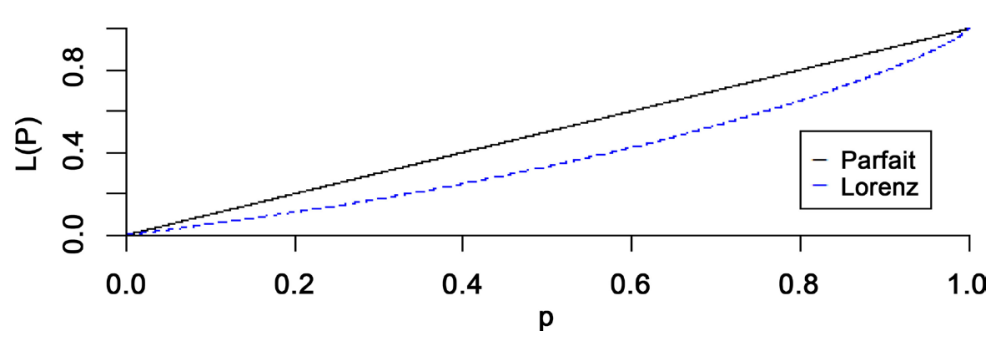

Figure 12. Lorenz curve of the $i B o x x$ portfolio.

Table 4. The computational result of the $i B o x x$ portfolio.

\begin{tabular}{cl}
$H H I$ & $0.07 \%$ \\
$H K I$ & $0.08 \%$ \\
$V a R$ & $6.42 \%$ \\
$E C$ & $5.87 \%$ \\
$G A$ & $0.07 \%$ \\
Approximate $G A$ & $0.08 \%$ \\
\hline
\end{tabular}

\footnotetext{
${ }^{15}$ Moody’s Investor Service, 2010.
} 

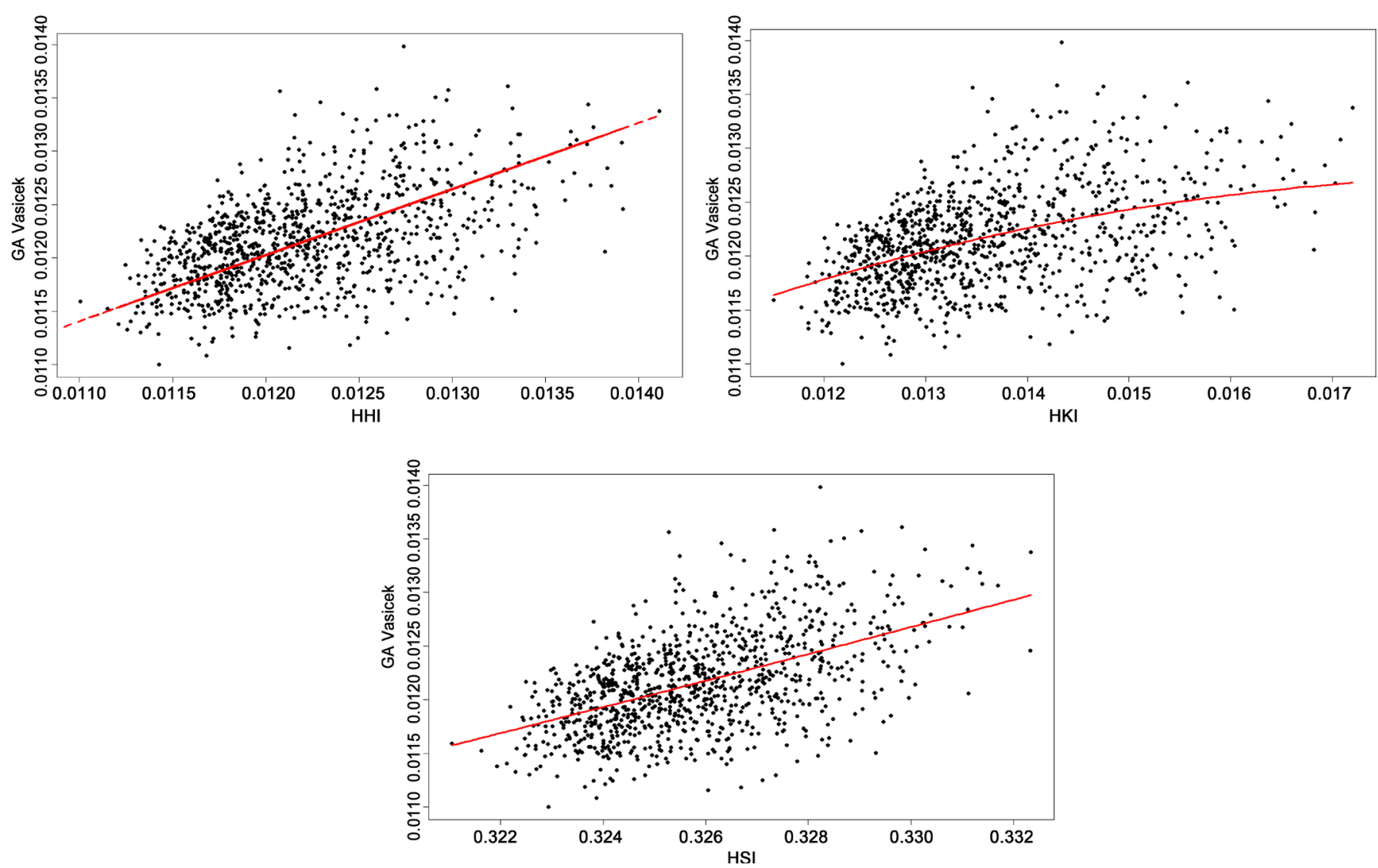

Figure 13. The regression of $G A$ on indexes.

Table 5. The concentration measure recapitulative of the credit portfolio.

\begin{tabular}{rlc}
\hline$H H I P$ & $1.17 \%$ \\
$H K I P$ & $1.25 \%$ \\
$H S I P$ & $32.42 \%$ \\
$V a R P$ & $6.22 \%$ \\
$E C P$ & $5.71 \%$ \\
$G A(H H I)$ & $1.183 \%$ \\
$G A(H K I)$ & $1.192 \%$ \\
$G A(H S I)$ & $1.195 \%$ \\
\hline
\end{tabular}

There is a concentration risk rather important consideration at the $G A$, as it increases the costs in terms of provision approximately $21 \%$. This result is consistent with the $H S I$ index, unlike the $H H I$ and $H K I$ indexes.

\section{Conclusion}

This paper is dedicated, firstly, to model the name concentration under the Add-On approach; secondly, to approximate the $G A$ using the concentration indexes. We established tests to find the relation between the $G A$ and the indexes. These approximations 
allow us some simplification of the $G A$ formula. As application, we chose the $i B o x x$ composition as the credit portfolio.

These tests on the $G A$ approximation enabled us to make the relation between the $A d-H o c$ and the Add-On. We retained the regression between the $G A$ and concentration indexes. Furthermore, the $H S I$ index gave a more consistent measurement of portfolios with a small number of exposures.

However, these approximations can be used to simplify the GA calculation under the sector concentration. Indeed, the formulation of $G A$ is more complex in the sector concentration than the name concentration.

\section{References}

Gourieroux, C., Laurent, J. P., \& Scaillet, O. (2000). Sensitivity Analysis of Values at Risk. Journal of Empirical Finance, 7, 225-245. https://doi.org/10.1016/S0927-5398(00)00011-6

Gordy, M., \& Lutkebohmert, E. (2007). Granularity Adjustment for Basel II. Discussion Paper Series 2: Banking and Financial Studies, Deutsche Bundesbank.

Gordy, B., \& Lutkebohmert, E. (2013). Granularity Adjustment for Regulatory Capital Assessment. International Journal of Central Banking, 9, 33-71.

Hannah, L., \& Kay, J. A. (1977). Concentration in Modern Industry. London: Mac Millan Press.

Herfindahl, O. (1950). Concentration in the U.S. Steel Industry. Dissertation, Columbia University.

Hirschmann, A. (1964). The Paternity of an Index. American Economic Review, 54, 761.

Lutkebohmert, E. (2009). Concentration Risk in Credit Portfolios. Berlin: Springer.

Martin, R., \& Wilde, T. (2002). Unsystematic Credit Risk. Risk Magazine, 15, 123-128.

Merton, R. (1974). On the Pricing of Corporate Debt: The Risk Structure of Interest Rates. The Journal of Finance, 29, 449-470. https://doi.org/10.1111/j.1540-6261.1974.tb03058.x

Slime, B., \& Hammami, M. (2016). Concentration Risk: The Comparison of the Ad-Hoc Approach Indexes. Journal of Financial Risk Management, 5, 43-56.

Vasicek, O. A. (1987). Probability of Loss on Loan Portfolio. San Francisco: KMV Corporation.

Wilde, T. (2001). Probing Granularity. Risk Magazine, 14, 103-106.

Credit Suisse Financial Products (1997). Credit Riskt: A Credit Risk Management Framework. London. 


\section{Annexes}

\section{- The Vasicek model:}

In 1987, Vasicek used the Merton model (1974) to modeling relations between the default events to get the assessment of the credit risk. We denote $\lambda_{i}$ as the liability of the borrower $i$. The asset value of this borrower with a giving time $t$ follows a geometric Brownian motion and verifies the following stochastic differential equation (SDE):

$$
\mathrm{d} V_{i, t}=V_{i, t}\left(\mu_{i} \mathrm{~d} t+\sum_{k=1}^{m} \sigma_{i, k} \mathrm{~d} W_{k, t}+\eta_{i} \mathrm{~d} B_{i, t}\right)
$$

With $\mu_{i}, \sigma_{1}, \cdots, \sigma_{m}, \eta_{i}$ are constant and $W_{1, t}, \cdots, W_{m, t}, B_{i, t}$ is an Independent Brownian motion. $W_{k, t}, k=1, \cdots, m$ represent the macroeconomic component (systematic risk) and $B_{i, t}$ is the specific factor (idiosyncratic risk). The Black \& Scholes theory with a one year horizon gives us the solution of the SDE:

$$
V_{i, 1}=V_{i, 0} \exp \left(\mu_{i}+\sum_{k=1}^{m}\left(\sigma_{i, k} X_{k}-\frac{1}{2} \sigma_{i, k}^{2}\right)+\eta_{i} \epsilon_{i}-\frac{1}{2} \eta_{i}^{2}\right)
$$

where $X_{1}, \cdots, X_{m}, \epsilon_{i}$ are i.i.d (independent and identically distributed) and follow a Gaussian distribution.

The model supposes that default variables $D_{i}$ are Bernoulli:

$$
D_{i}= \begin{cases}1 & \text { si } V_{i, 1}<\lambda_{i} \\ 0 & \text { si } V_{i, 1} \geq \lambda_{i}\end{cases}
$$

Indeed, the default probability is equal to:

$$
\begin{aligned}
P D_{i} & =\mathbb{P}\left(V_{i, 1}<\lambda_{i}\right)=\mathbb{P}\left(V_{i, 0} \exp \left(\mu_{i}+\sum_{k=1}^{m}\left(\sigma_{i, k} X_{k}-\frac{1}{2} \sigma_{i, k}^{2}\right)+\eta_{i} \epsilon_{i}-\frac{1}{2} \eta_{i}^{2}\right)<\lambda_{i}\right) \\
& =\mathbb{P}\left(\sum_{k=1}^{m} \sigma_{i, k} X_{k}+\eta_{i} \epsilon_{i}<\ln \left(\frac{\lambda_{i}}{V_{i, 0}}\right)+\sum_{k=1}^{m} \frac{1}{2} \sigma_{i, k}^{2}+\frac{1}{2} \eta_{i}^{2}-\mu_{i}\right) \\
& =\mathbb{P}\left(\frac{\sum_{k=1}^{m} \sigma_{i, k} X_{k}+\eta_{i} \epsilon_{i}}{\sqrt{\sum_{k=1}^{m} \sigma_{i, k}^{2}+\eta_{i}^{2}}}<\frac{\ln \left(\frac{\lambda_{i}}{V_{i, 0}}\right)+\sum_{k=1}^{m} \frac{1}{2} \sigma_{i, k}^{2}+\frac{1}{2} \eta_{i}^{2}-\mu_{i}}{\sqrt{\sum_{k=1}^{m} \sigma_{i, k}^{2}+\eta_{i}^{2}}}\right) \\
& =\Phi\left(\frac{\ln \left(\frac{\lambda_{i}}{V_{i, 0}}\right)+\sum_{k=1}^{m} \frac{1}{2} \sigma_{i, k}^{2}+\frac{1}{2} \eta_{i}^{2}-\mu_{i}}{\sqrt{\sum_{k=1}^{m} \sigma_{i, k}^{2}+\eta_{i}^{2}}}\right)
\end{aligned}
$$

Therefore, the borrower is in default when:

$$
\frac{\sum_{k=1}^{m} \sigma_{i, k} X_{k}+\eta_{i} \epsilon_{i}}{\sqrt{\sum_{k=1}^{m} \sigma_{i, k}^{2}+\eta_{i}^{2}}}<\frac{\ln \ln \left(\frac{\lambda_{i}}{V_{i, 0}}\right)+\sum_{k=1}^{m} \frac{1}{2} \sigma_{i, k}^{2}+\frac{1}{2} \eta_{i}^{2}-\mu_{i}}{\sqrt{\sum_{k=1}^{m} \sigma_{i, k}^{2}+\eta_{i}^{2}}}
$$


If we set: $\rho_{i}=\frac{\sum_{k=1}^{m} \sigma_{i, k}^{2}}{\sum_{k=1}^{m} \sigma_{i, k}^{2}+\eta_{i}^{2}}$ et $\alpha_{i, k}=\frac{\sigma_{i, k}}{\sqrt{\sum_{k=1}^{m} \sigma_{i, k}^{2}}}$

We get: $\frac{\sum_{k=1}^{m} \sigma_{i, k} Z_{k}+\eta_{i} \epsilon_{i}}{\sqrt{\sum_{k=1}^{m} \sigma_{i, k}^{2}+\eta_{i}^{2}}}=\sqrt{\rho_{i}} \alpha_{i}^{\prime} \boldsymbol{X}+\sqrt{1-\rho_{i}} \epsilon_{i}$

With $\boldsymbol{\alpha}_{i}^{\prime}=\left(\alpha_{i, 1}, \cdots, \alpha_{i, m}\right)$ and $\boldsymbol{X}^{\prime}=\left(X_{1}, \cdots, X_{m}\right)$

The default condition becomes:

$$
D_{i}=\left\{\begin{array}{l}
1 \text { si } \sqrt{\rho_{i}} \alpha_{i}^{\prime} \boldsymbol{X}+\sqrt{1-\rho_{i}} \epsilon_{i}<\Phi^{-1}\left(P D_{i}\right) \\
0 \text { si } \sqrt{\rho_{i}} \alpha_{i}^{\prime} \boldsymbol{X}+\sqrt{1-\rho_{i}} \epsilon_{i} \geq \Phi^{-1}\left(P D_{i}\right)
\end{array}\right.
$$

With $\Phi^{-1}\left(P D_{i}\right)=\frac{\ln \left(\frac{\lambda_{i}}{V_{i, 0}}\right)+\sum_{k=1}^{m} \frac{1}{2} \sigma_{i, k}^{2}+\frac{1}{2} \eta_{i}^{2}-\mu_{i}}{\sqrt{\sum_{k=1}^{m} \sigma_{i, k}^{2}+\eta_{i}^{2}}}$

Then, we conclude that:

$$
\rho_{i} \in[0,1], \sum_{k=1}^{m} \alpha_{i, k}^{2}=1, \sqrt{\rho_{i}} \alpha_{i}^{\prime} \boldsymbol{X}+\sqrt{1-\rho_{i}} \epsilon_{i} \sim N(0,1)
$$

The Vasicek model use one systematic factor $\boldsymbol{X}=X$. The default probability of some borrower conditionally to this factor is equal to:

$$
\begin{aligned}
P D_{i}(x) & =\mathbb{P}\left(D_{i}=1 \mid X=x\right)=\mathbb{P}\left(\sqrt{\rho_{i}} \alpha_{i}^{\prime} \boldsymbol{X}+\sqrt{1-\rho_{i}} \epsilon_{i}<\Phi^{-1}\left(P D_{i}\right) \mid X=x\right) \\
& =\mathbb{P}\left(\sqrt{\rho_{i}} x+\sqrt{1-\rho_{i}} \epsilon_{i}<\Phi^{-1}\left(P D_{i}\right)\right)=\mathbb{P}\left(\epsilon_{i}<\frac{\Phi^{-1}\left(P D_{i}\right)-\sqrt{\rho_{i}} x}{\sqrt{1-\rho_{i}}}\right)
\end{aligned}
$$

We can deduce that $P D_{i}(x)=\Phi\left(\frac{\Phi^{-1}\left(P D_{i}\right)-\sqrt{\rho_{i}} x}{\sqrt{1-\rho_{i}}}\right)$

Giving these results and under the assumption that borrowers loss are independent. The loss rate of the whole portfolio is:

$$
\left.L_{N}=\sum_{i=1}^{N} s_{i} L G D_{i} \mathbb{1}_{\left\{\sqrt{\rho_{i}} \alpha_{i}^{\prime} X+\sqrt{1-\rho_{i}} \epsilon_{i}<\Phi^{-1}\left(P D_{i}\right)\right.}\right\}
$$

We can obtain the expected loss conditionally to the systematic factor under the assumption that the loss giving default $L G D_{i}$ and the default event $D_{i}=\mathbb{1}_{\left\{\sqrt{\rho_{i}} \alpha_{i}^{\prime} X+\sqrt{1-\rho_{i}} \epsilon_{i}<\Phi^{-1}\left(P D_{i}\right)\right\}}$ are independent:

$$
\mathbb{E}\left[L_{N} \mid X\right]=\sum_{i=1}^{N} s_{i} L G D_{i} \Phi\left(\frac{\Phi^{-1}\left(P D_{i}\right)-\sqrt{\rho_{i}} \alpha_{i}^{\prime} \boldsymbol{X}}{\sqrt{1-\rho_{i}}}\right)
$$

We can use the Monte Carlo simulation on the systematic factor to compute this value.

- The Credit Risk+ model:

The Credit Riskt model was had developed by Credit Suisse Financial Products (CSFP). This model is the one of most used in the IRB Approach and he is one of reduced form models. The default rate is a stochastic variable and the default variable 
follows the Bernoulli distribution:

$$
D_{i}=\left\{\begin{array}{l}
1 \text { si l'emprunteur i fait défaut à } \mathrm{T} \\
0 \text { autrement }
\end{array}\right.
$$

Credit Risk+ supposes that default probabilities are hazardous and systematic factors follow the Gamma distribution with the following function density:

$$
\Gamma_{\alpha, \beta}(x)=\frac{1}{\beta^{\alpha} \Gamma(\alpha)} \mathrm{e}^{-x} x^{\alpha-1} \text {, pour } x \geq 0 \text { et } \beta=\frac{1}{\alpha}
$$

With $\mathbb{E}[X]=\alpha \beta=1$ and $\mathbb{V}[X]=\alpha$

In the case that the default frequency $\tilde{D}_{i}$ follows the Poisson distribution with $P D_{i}$ as intensity, we get:

$$
\mathbb{P}\left(\tilde{D}_{i}=k \mid X=x\right)=\frac{\left(P D_{i} \times x\right)^{k}}{k !} \exp \left(-P D_{i} \times x\right), k=0,1,2, \cdots
$$

The default variable and the default frequency meet with the following relation $D_{i}=\mathbb{1}_{\left\{\tilde{D}_{i} \geq 1\right\}}$. Therefore, the conditional probability is defined as:

$$
\begin{aligned}
P D_{i}(X) & =\mathbb{P}\left(D_{i}=1 \mid X\right) \\
& =\mathbb{P}\left(\tilde{D}_{i} \geq 1 \mid X\right) \\
& =1-\mathbb{P}\left(\tilde{D}_{i}=0 \mid X\right) \\
& =1-\exp \left(-P D_{i} \times X\right)
\end{aligned}
$$

Submit or recommend next manuscript to SCIRP and we will provide best service for you:

Accepting pre-submission inquiries through Email, Facebook, LinkedIn, Twitter, etc.

A wide selection of journals (inclusive of 9 subjects, more than 200 journals)

Providing 24-hour high-quality service

User-friendly online submission system

Fair and swift peer-review system

Efficient typesetting and proofreading procedure

Display of the result of downloads and visits, as well as the number of cited articles

Maximum dissemination of your research work

Submit your manuscript at: http://papersubmission.scirp.org/

Or contact jfrm@scirp.org 